\title{
Binding Geometry of Inclusion Complex as a Determinant Factor for Aqueous Solubility of the Flavonoid/ $\beta$-Cyclodextrin Complexes Based on Molecular Dynamics Simulations
}

\author{
Youngjin Choi, ${ }^{1}$ Jonghyun Lee, ${ }^{2}$ Kum Won Cho,${ }^{3}$ Suntae Hwang, ${ }^{4}$ Karpjoo Jeong, ${ }^{2,5}$ and Seunho Jung ${ }^{1,5, *}$ \\ ${ }^{1}$ Department of Microbial Engineering \& ${ }^{5}$ Department of Advanced Technology Fusion, Bio/Molecular Informatics Center, \\ Konkuk University, Seoul 143-701, Korea. E-mail: shjung@konkuk.ac.kr \\ ${ }^{2}$ College of Information and Communication, Konkuk University, Seoul 143-701, Korea \\ ${ }^{3}$ Korea Institute of Science and Technology Information, Daejeon 305-806, Korea \\ ${ }^{4}$ Department of Computer Science, Kookmin University, Seoul 136-702, Korea \\ Received June 8, 2005
}

\begin{abstract}
A computational study based on molecular dynamics (MD) simulations was performed in order to explain the difference in aqueous solubilities of two flavonoid $/ \beta$-cyclodextrin $(\beta$-CD) complexes, hesperetin $/ \beta$-CD and naringenin $/ \beta$-CD. The aqueous solubility of each flavonoid/ $\beta$-CD complex could be characterized by complexwater interaction not by flavonoid-CD interaction. The radial distribution of water around each inclusion complex elucidated the difference of an experimentally observed solubility of each flavonoid/ $\beta$-CD complex. The analyzed results suggested that a bulky hydrophobic moiety $\left(-\mathrm{OCH}_{3}\right)$ of $B$-ring of hesperetin nearby primary rim of $\beta$-CD was responsible for lower aqueous solubility of the hesperetin/ $\beta$-CD complex.
\end{abstract}

Key Words : Aqueous solubility, Cyclodextrin, Flavonoid, Inclusion complex, Molecular dynamics simulations

\section{Introduction}

Aqueous solubility of biologically active compounds has been of great interest to pharmaceutical research areas. ${ }^{1}$ The solubility is an important chemical property that influences a release or transport of drugs in the human body and that is a determinant of environmental fate of chemicals in nature. ${ }^{2} \mathrm{~A}$ class of poorly insoluble drugs should be chemically processed via covalent or non-covalent modification to enhance the aqueous solubility and availability.

The flavonoids are members of natural compounds produced by the green plant cells. They readily participate in biological process such as radical scavenge, electron transfer, and signal transduction. ${ }^{3}$ They are potential medical applicants for hypertension, cancer, inflammation, and cardiovascular disease. ${ }^{4,5}$ But easy-use of the flavonoid has been regarded as a long-standing problem for many people because its aqueous solubility is very low. Cyclodextrins (CDs) and their derivatives are one of the most important solubilizing agents for various drugs, food additives, and other hydrophobic molecules. ${ }^{6,7}$ They form a host-guest type inclusion complex with small organic molecules including flavonoid and then lead to changes in the solubility of the guest molecules. Recent study by Tommasini et al. ${ }^{8}$ for the inclusion complex of flavonoids with $\beta$-CD revealed that the aqueous solubility of naringenin was much increased by $\beta$ $\mathrm{CD}$ compared with hesperetin by $\beta \mathrm{CD}$ at room-temperature.

In view of limited amount of the structural information available, computational methods can provide a useful means to develop theoretical model for aqueous solubility of flavonoid/ $\beta$-CD complexes. This paper describes the thermodynamic and structural basis for the flavonoid/ $\beta-\mathrm{CD}$ inclusion complexes with regard to the origin of solubility difference between hesperetin/ $\beta$-CD and naringenin $/ \beta$-CD complexes. The solubilizing ability of $\beta$-CD for each flavonoid was explained by a distribution of water molecules around each flavonoid/ $\beta$-CD complex. The binding geometry analysis from the 6-ns MD simulations provided us a detailed picture of flavonoid-CD interaction and a clear answer for why hesperetin/ $\beta$-CD complex showed lower aqueous solubility compared with those of naringenin $/ \beta-\mathrm{CD}$ complex.

\section{Results and Discussion}

Figure 1 is a molecular representation of the naringenin and hesperetin concerning with a potential interaction site with water. Four-interaction sites were defined along by the oxygen atoms of the flavonoids. Both flavonoid compounds showed a similar geometry each other except a $D$-site. The hesperetin has an additional methoxy $\left(-\mathrm{OCH}_{3}\right.$, methyl cap) functional group at para-position of aromatic $B$-ring and its hydroxyl group was substituted at meta-position. A difference in interaction mode between each flavonoid $/ \beta-C D$ complex and solvent water was established with MD simulations. Figure 2 is a thermodynamic-behavior of naringenin/ $\beta$-CD complex at different temperature ranges. The value of experimentally determined association con$\operatorname{stant}^{8}$ (Figure 2A) is decreased along with the increase of temperature. This decreasing pattern indicates that solubilization process of naringenin $/ \beta$-CD complex is an exo- 

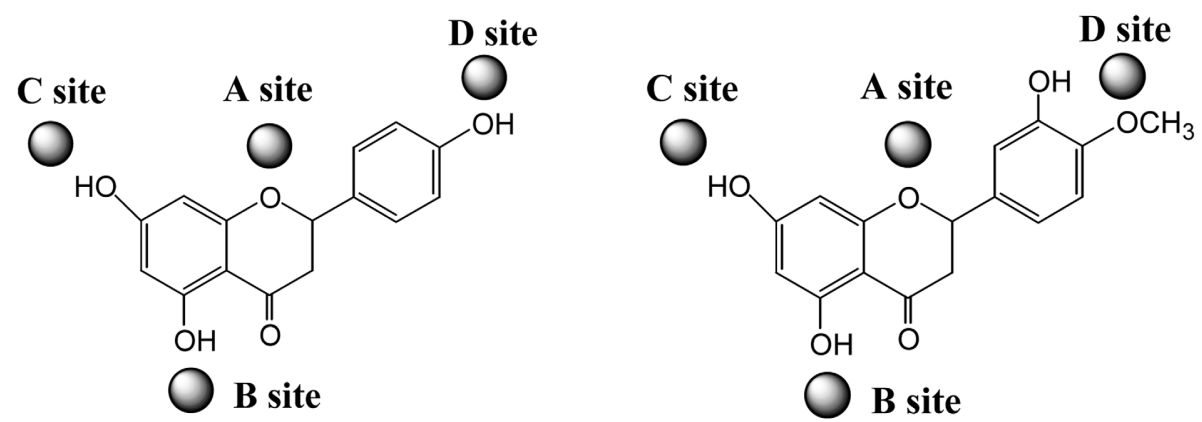

Figure 1. Schematic representations of two-dimensional structures of naringenin (left) and hesperetin (right). Four interaction sites were defined according to location of oxygen atoms of each flavonoid compound.
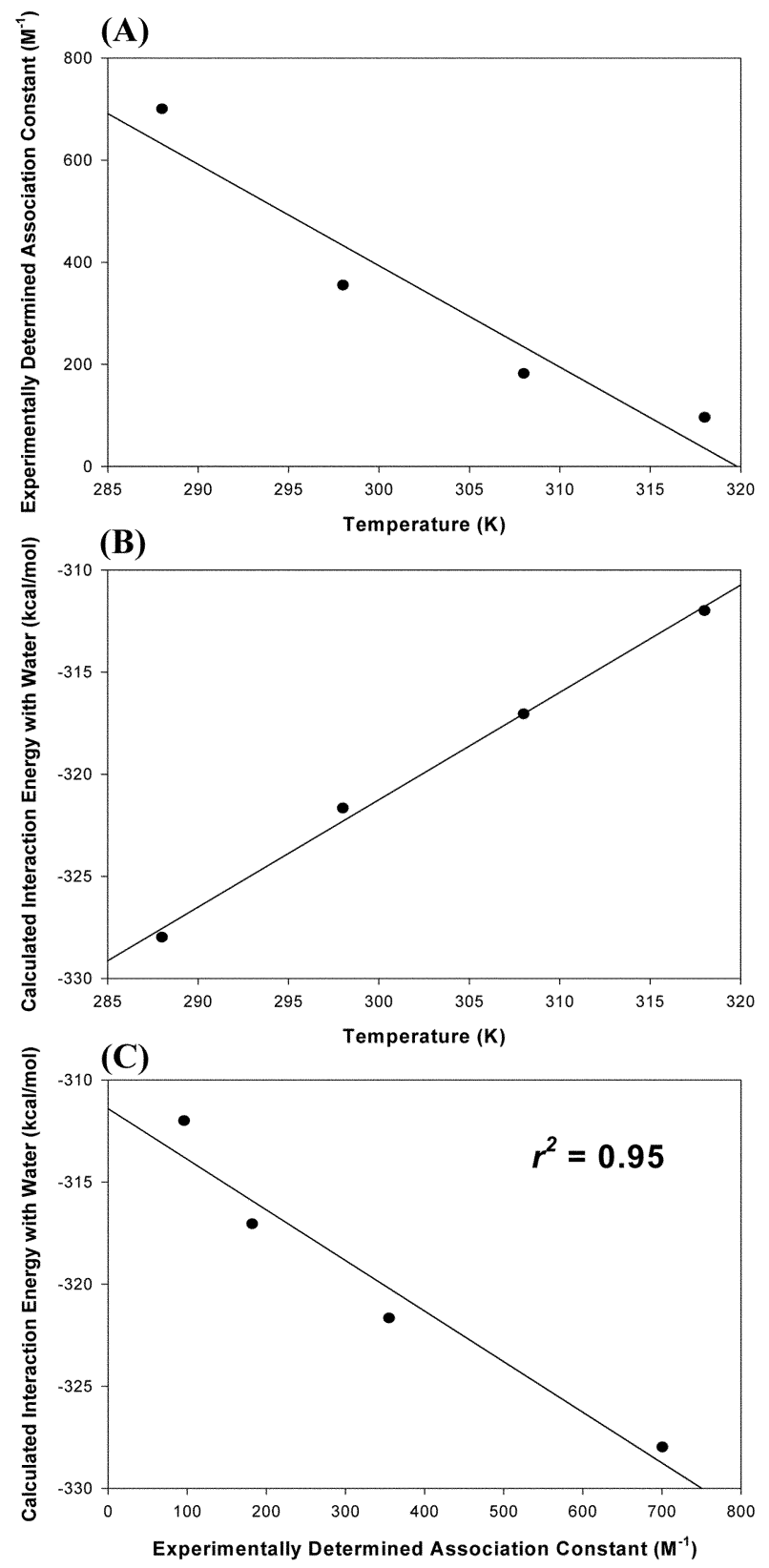

Figure 2. The experimentally determined association constants of naringenin $/ \beta-\mathrm{CD}$ complex (A), calculated interaction energy between the complex and water at different temperatures (B) and the correlation between calculated and experimentally determined results $(\mathrm{C})$.

thermic reaction. Such a behavior was well-reproduced with computational results in terms of intermolecular energy between the inclusion complex and water during the MD simulations (Figure 2B). The interaction energy was decreased along with increase of temperature like a case of experimental association constant. This consistence $\left(r^{2}=\right.$ $0.95)$ between experimental association constant and computational interaction energy evidenced a suitability of our computational method for model system (Figure 2C).

Detailed analyses for the MD-trajectories of each complex were performed to enhance our understanding for the solubilization process of naringenin $/ \beta-\mathrm{CD}$ and hesperetin $/ \beta$ $\mathrm{CD}$ complexes. Figure 3 is the interaction energy profile for each flavonoid $/ \beta$-CD complex during MD simulations. The intermolecular energy between naringenin and $\beta-\mathrm{CD}$ was $-19.27 \mathrm{kcal} / \mathrm{mol}$ and those of hesperetin and $\beta-\mathrm{CD}$ was $-23.56 \mathrm{kcal} / \mathrm{mol}$. (Figure $3 \mathrm{~A}, \mathrm{C}$ ) That means the hesperetin molecule forms a more stable complex with $\beta$-CD than the naringenin $/ \beta$-CD complex. This result seems to be contradictory to the experimental observation that naringenin $/ \beta$ $\mathrm{CD}$ complex has higher association constant than those of hesperetin/ $\beta$-CD complex from the aqueous solubility measurement. That contradiction between experimental and computational results could be solved by using an interaction energy profile between water and whole inclusion complex (Figure 3B, D). The intermolecular energy between naringenin $/ \beta-\mathrm{CD}$ and water was $-321.66 \mathrm{kcal} / \mathrm{mol}$ and those of hesperetin $/ \beta$-CD and water was $-316.16 \mathrm{kcal} / \mathrm{mol}$. The lower interaction energy between naringenin $/ \beta$-CD and water is well-consistent with the experimental observation regarding the higher association constant and aqueous solubility of the naringenin $/ \beta-\mathrm{CD}$ complex. This fact indicated that the solubilization processes of the flavonoids by $\beta$-CD should be analyzed mainly by the whole inclusion complex-water interaction not by the host CD-guest flavonoid interaction. The association constants, interaction energies, and hydration numbers are summarized in Table 1. The naringenin $/ \beta$-CD complex was heavily solvated by water molecules than hesperetin/ $\beta$-CD complex. The results of MD simulations could finely explain the higher aqueous solubility of naringenin/ $\beta$-CD complex compared with hesperetin/ $\beta$-CD complex.

The different aqueous solubility of naringenin and 

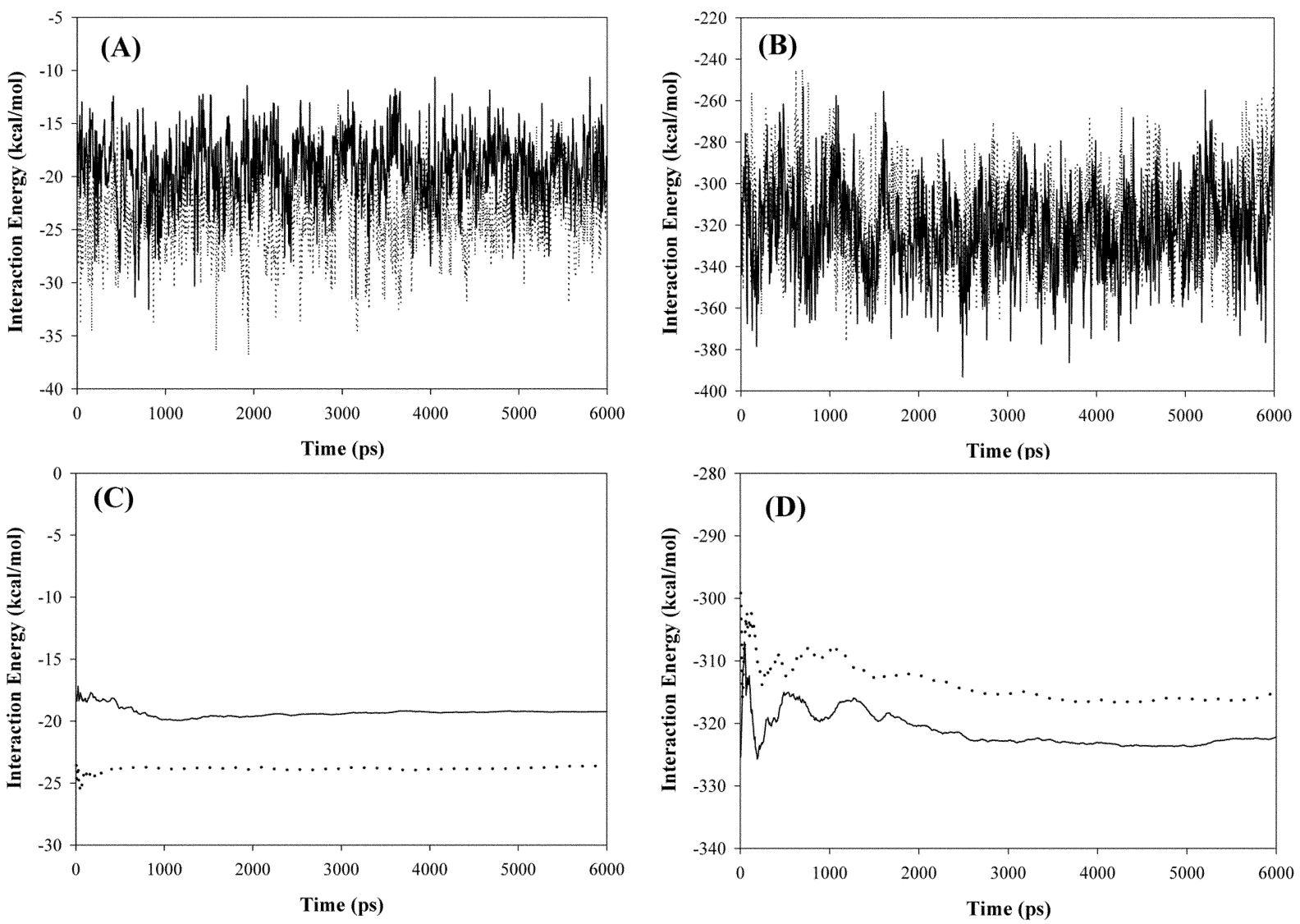

Figure 3. Interaction energy profiles of each flavonoid- $\beta-\mathrm{CD}(\mathrm{A})$ and whole complex-water (B) during 6-ns MD simulations. The naringenin $/ \beta$-CD complex (solid line) showed lower interaction energy with water than those of hesperetin/ $\beta$-CD complex (dashed line). A stable profile of cumulative interaction energies $(C, D)$ indicated that the MD production phases were fully equilibrated.

Table 1. Association constants $\left(K_{\mathrm{c}}\right)$, interaction energy $(\Delta E)$, hydration number $\left(N_{H}\right)$ of complexes naringenin/ $\beta$-CD and hesperetin $/ \beta-\mathrm{CD}$ at room temperature

\begin{tabular}{cccccc}
\hline Complex & & $K_{c}\left(\mathrm{M}^{-1}\right)$ & $\Delta E_{C D}$ & $\Delta E_{\text {Water }}$ & $N_{H}$ \\
\cline { 5 - 7 } \cline { 5 - 6 } Naringenin $/ \beta-C D$ & & 355.06 & -19.27 & -321.66 & 3.07 \\
Hesperetin $/ \beta-C D$ & & 234.48 & -23.56 & -316.16 & 2.99 \\
\hline
\end{tabular}

hesperetin in the presence of $\beta$-CD was due to the hydrational states of both flavonoids/ $\beta$-CD complexes. Different hydration pattern of each flavonoid/ $\beta$-CD complex was investigated by a radial distribution function (RDF) of water around oxygen atoms of each flavonoid within $\beta$-CD (Figure 4). The RDF value gives the information for the probable distribution of water molecules around specific atoms or molecules, where the RDF was calculated for the water around 4-interaction sites of each flavonoid within $\beta$ CD. An interatomic RDF analysis shows that distributions of water are dissimilar to each other and revealed that $A$-site of each flavonoid was included within a cavity of $\beta$-CD. Thus, the $A$-site is expected to show a very weak interaction with water at both naringenin $/ \beta-\mathrm{CD}$ and hesperetin/ $\beta-\mathrm{CD}$ complexes and the hydration shell peak around $A$-site of each flavonoid was not observed from this RDF analysis at all (Figure 4A). However, the RDF of $B$-site of naringenin/ $\beta$ -
CD was obtained with a well-defined first hydration shell of which there is a density peak at $2.6 \AA$ with a peak density of about 2.11. Similarly, the RDF calculated for $C$-site of naringenin/ $\beta$-CD displays a density peak at $2.6 \AA$ and a peak density of about 2.25 . These water distributions of $B$ - and $C$ sites of naringenin $/ \beta$-CD were almost identical with those of hesperetin $/ \beta$-CD complex. The hydration peak each for $B-$ and $C$-site of hesperetin/ $\beta$-CD complex was observed at 2.6 $\AA$ with the density of 2.04 and 2.27 , respectively (Figure 4B, $\mathrm{C})$. The $B$ - and $C$-sites of the each flavonoid protrude to the wide secondary rim of $\beta$-CD and they are able to interact with water molecules with a long-lived hydration structure. Since the hydroxyl group of both flavonoids nearby the secondary rim of $\beta$-CD can make a strong interaction with water molecules, RDF values around $B$ - and $C$-sites of the flavonoid $/ \beta$-CD complexes could not explain the reason for the solubility difference between naringenin/ $\beta-\mathrm{CD}$ and hesperetin $/ \beta-\mathrm{CD}$ complexes. However, the RDF value around $D$-sites showed an apparent difference between these complexes (Figure 4D). The hydration peak for $D$-site of each flavonoid $/ \beta$-CD complex was observed at $2.6 \AA$ with the density of 2.09 for the naringenin/ $\beta$-CD and at $3.2 \AA$ with the density of 0.75 for hesperetin $/ \beta-C D$ complex, respectively. Since the $D$-sites of both flavonoids are located on nearby the primary rim of $\beta-\mathrm{CD}$, they are directly 

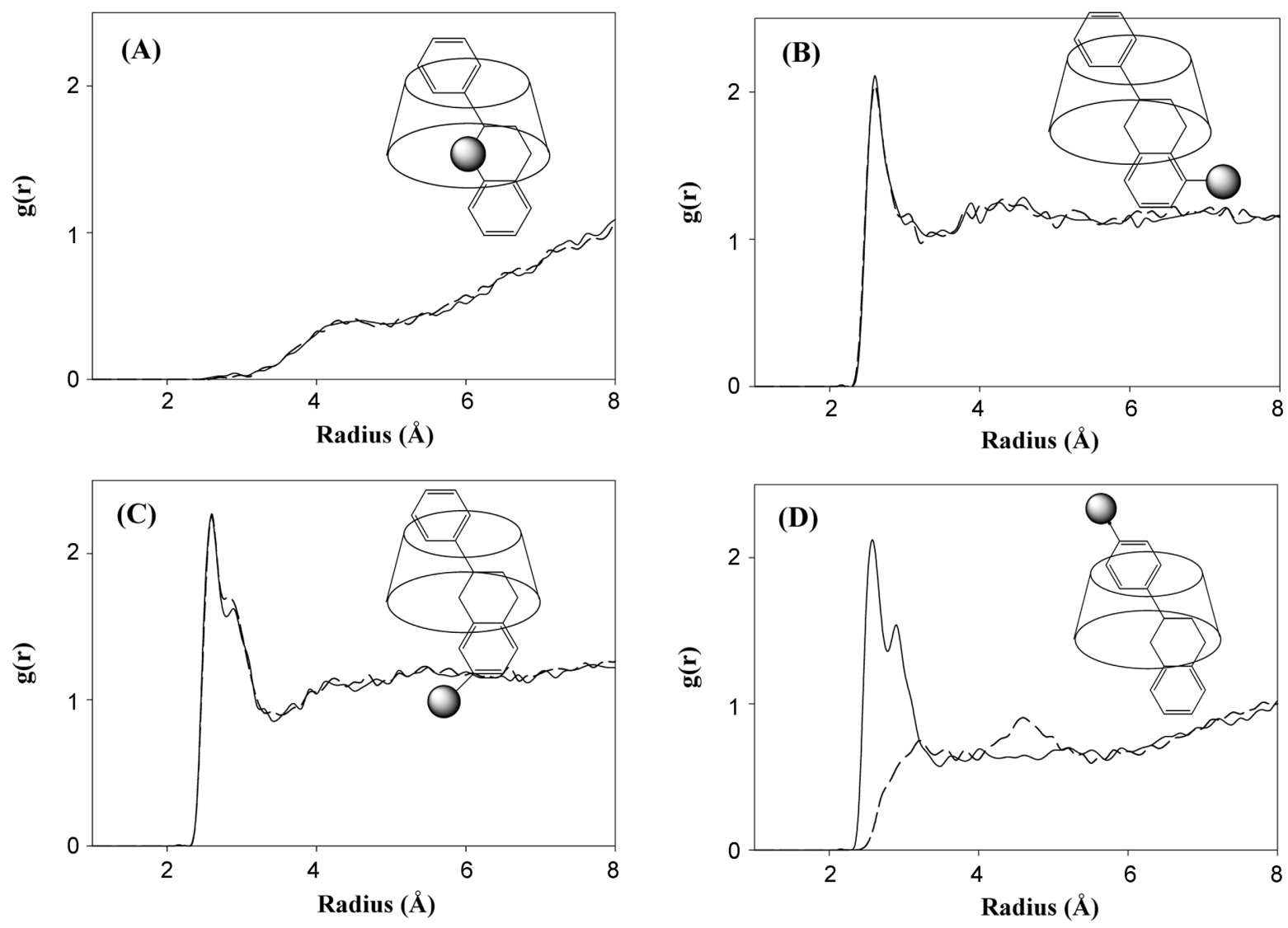

Figure 4. Radial distribution function (RDF) of water and oxygen atoms of naringenin $/ \beta$-CD complex (solid line) and hesperetin/ $\beta$-CD complex (dashed line). Each RDF around oxygen atoms of $A-, B-, C$-, and $D$-sites was plotted at separate panel. A dramatic difference in hydration behavior of naringenin/ $\beta$-CD complex and hesperetin $/ \beta$-CD complex was observed at $D$-site.

coupled with the differences in hydration status between theses two flavonoid $/ \beta$-CD complexes. The narrow primary rim of the $\beta$-CD can restrict the free migration of solvent water molecules around the $D$-sites of each flavonoid. We think that this molecular environment adjacent to the $D$-site of flavonoid compounds determines the aqueous solubility of the flavonoid $/ \beta$-CD complexes.

The docked conformations with the lowest-energy of each flavonoid $/ \beta$-CD during the MD simulations give visual information on the binding geometries of the inclusion complexes (Figure 5). The $D$-site of naringenin/ $\beta$-CD was exposed to the neighboring water molecules directly; however, the $D$-site of hesperetin was geometrically blocked by a hydrophobic bulky methyl group. This hydrophobic "cap" of hesperetin seems to be responsible for the lower aqueous solubility of the hesperetin $/ \beta$-CD complex. The binding geometry analysis for the solubilization mechanism proved that the exposed solvent-accessible moiety of guest flavonoid from the cavity of $\beta$-CD was a decisive factor to determine aqueous solubility of the flavonoid $/ \beta$-CD complexes. In this respect, the geometric design of docked conformation for the inclusion complex would be highly recommended to gain a desirable aqueous solubility of flavonoid compounds based on inclusion complex technology.

\section{Methods of Computation}

Construction of the molecular models and protocol of MC docking simulations. The starting configuration of the $\beta-\mathrm{CD}$ for MC simulations was taken from the X-ray crystal structure. The InsightII/Builder module (version 2000, Accelrys Inc. San Diego, USA) was used to generate missing hydrogen atoms for the $\beta$-CD used in the simulations. The atomic coordinates of the hesperetin and naringenin were obtained from Sci-Finder. Their twodimensional molecular structures are depicted in Figure 1. All simulations were performed using a general molecular modeling program, CHARMM $^{9}$ (version 28b2), with a parm 22 all-atom force field. The parameter values for the $\beta$ $\mathrm{CD}$ were modified according to a revised carbohydrate parameter set (carbohydrate solution force field-CSFF ${ }^{10}$ ) of the CHARMM. The MC docking simulations were performed using a "MC" module of CHARMM. The short-range nonbonded interactions were truncated with a $13-\AA$ cutoff. An implicit solvent water model was used with a distancedependent dielectric constant. The docking process was assumed to be a $1: 1$ interaction between $\beta$-CD and each flavonoid during the MC runs. The initial configuration of each host and guest molecule was positioned arbitrarily within a neighboring distance. Trials to a new configuration 

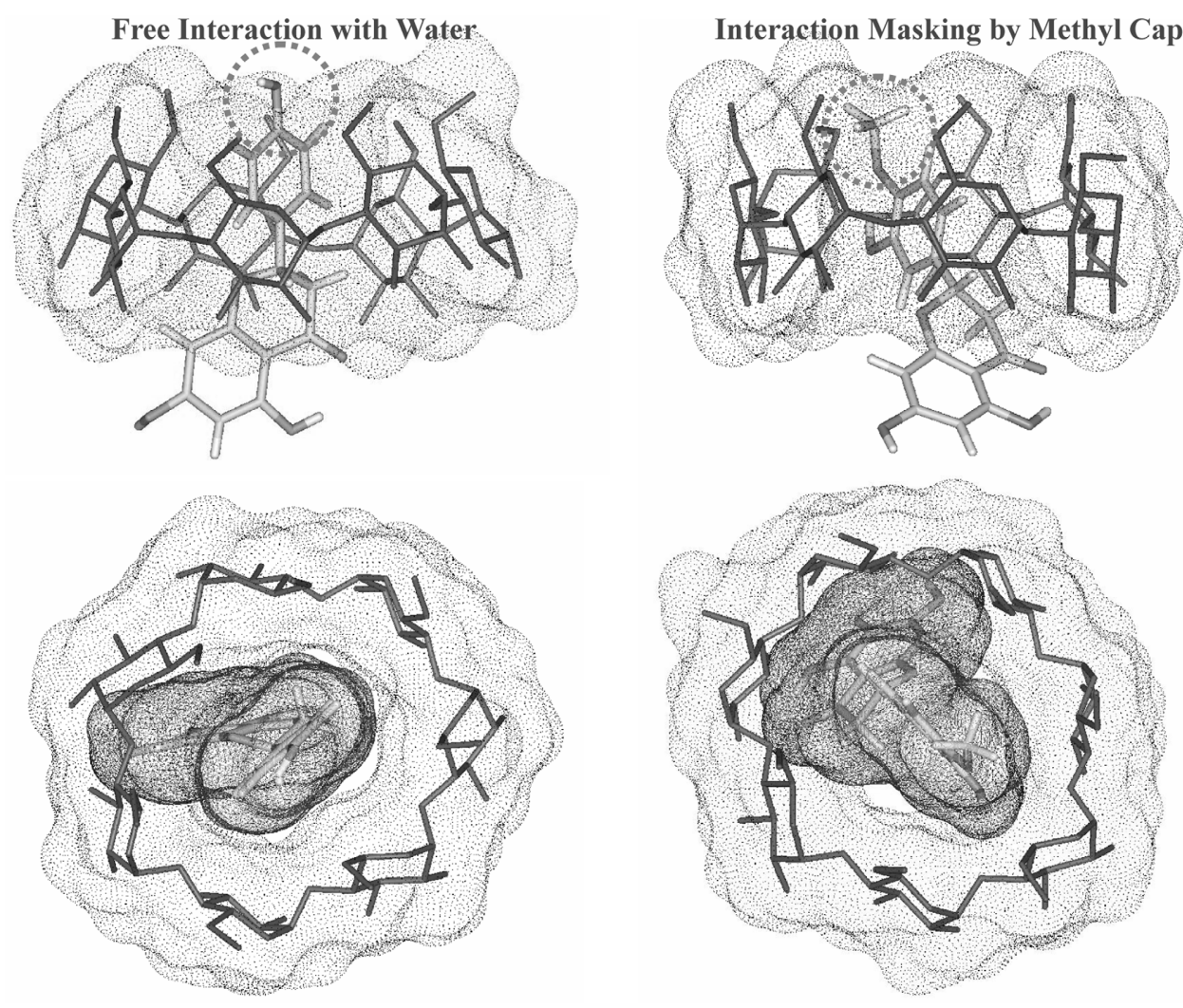

Side View

Top View

Figure 5. Side and Top views of MD-generated conformations for the naringenin/ $\beta$-CD (left) and hesperetin/ $\beta$-CD complex (right). Solventaccessible surface area of $\beta$-CD was represented as a dotted picture. (right). Relative orientations of the guest flavonoids for the $\beta$-CD cavities were maintained during the MD simulations. The oxygen atom of hesperetin on the primary rim of the $\beta$-CD was geometrically blocked from solvent water by hydrophobic methyl cap. Molecular surface rendering was performed with MD-generated conformations with the lowest energy using InsightII program.

were accomplished by changing each move set of a guest molecule. The MC move set for flexible docking was composed of rigid translations, rigid rotations, and rotations of freely rotatable dihedral angles of the guest. A single step consists of picking a random conformer, making a random move, minimizing the energy of a new conformer, and then checking the energy with a Metropolis ${ }^{11}$ criterion. This process uses a combined methodology consisting of Metropolis criterion for a global optimization and an energy minimization method for a local optimization. ${ }^{12}$ The $\beta$-CD was weakly fixed using a harmonic positional restraint of CHARMM to maintain backbone integrity. The MCminimized structures were saved every 20 steps for 20,000 trials. These MC processes produced various docked structures for each guest with $\beta$-CD.

Molecular dynamics simulations of the each flavonoid/ $\beta$-CD complex. The starting configurations of each falvonoid $/ \beta$-CD complex for the MD simulations in water were taken from the MC-docked conformations with the lowest-energy value. The geometries of these molecular models were fully optimized before MD runs. A TIP3P three-site rigid water ${ }^{13}$ model was used to solvate the complexes. Water molecules were removed if they were closer than $2.6 \AA$ to any heavy atoms of the complexes. In summary, each system was constructed using periodic boundary conditions with a cubic box of dimensions $30 \AA \times$ $30 \AA \times 30 \AA$, consisting of hesperetin/ $\beta$-CD complex and 926 water, or naringenin/ $\beta$-CD complex and 925 water molecules. The system was minimized by 1,000 steps of conjugate gradient, followed by Adopted Basis NewtonRaphson until the root-mean-square gradient was less than $0.001 \mathrm{kcal} / \mathrm{mol}$. The MD simulations were performed using the CHARMM 28b2 program in the isothermal-isobaric ensemble at 4-different temperature conditions ( $P=1 \mathrm{bar}, T$ $=288,298,308,318 \mathrm{~K})$. The particle mesh Ewald summation $^{14}$ method was used to treat the long-range electrostatic interactions. The bond lengths of water molecules were constrained with the SHAKE ${ }^{15}$ algorithm. The time step was $1.0 \mathrm{fs}$, and the non-bonded pair list was updated every 50 steps. The short-range non-bonded interactions were truncated with a $13-\AA$ cutoff. The temperature and pressure of the system was regulated using the Langevin piston method in conjunction with Hoover's thermostat. ${ }^{16}$ The system was gradually heated to each targeted temperature for $80 \mathrm{ps}$ and equilibrated for $1,000 \mathrm{ps}$ at this temperature. The production MD trajectory with one snapshot per 5 ps was collected for 5,000 ps.

Acknowledgements. This study was supported by the Korea Research Foundation (KRF2004-F00019) and 
partially supported by a grant of the MIC (Ministry of Information and Communication) through e-Science Project of KISTI (Korea Institute of Science and Technology Information). SDG.

\section{References}

1. Taskinen, J. Curr. Opin. Drug Discov. Dev. 2000, 3, 102.

2. Jorgenson, W. L.; Duffy, E. M. Adv. Drug Deliv. Rev. 2002, 54, 355.

3. Havsteen, B. H. Pharm. Therap. 2002, 96, 67.

4. Ren, W.; Qiao, Z.; Wang, H.; Zhu, L.; Zhang, L. Med. Res. Rev. 2003, 23, 519.

5. Zand, R. S. R.; Jenkins, D. J. A.; Diamandis, E. P. J. Chromatogr. B 2002, 777, 219.

6. Hedges, A. R. Chem. Rev. 1998, 98, 2035.

7. Uekama, K.; Hirayama, F.; Irie, T. Chem. Rev. 1998, 98, 2045.
8. Tommasini, S.; Raneri, D.; Ficarra, R.; Calabro, M. L.; Stancanelli, R.; Ficarra, P. J. Pharm. Biomed. Anal. 2004, 35, 379.

9. Brooks, B. R.; Bruccoleri, R. E.; Olafson, B. D.; States, D. J.; Swaminathan, S.; Karplus, M. J. Comput. Chem. 1983, 4, 187.

10. Kuttel, M.; Brady, J. W.; Naidoo, K. J. J. Comput. Chem. 2002, 23, 1236.

11. Metropolis, N.; Rosenbluth, A. W.; Rosenbluth, M. N.; Teller, A. H.; Teller, E. J. Chem. Phys. 1953, 21, 1087.

12. Caflisch, A.; Fischer, S.; Karplus, M. J. Comput. Chem. 1997, 18, 723.

13. Jorgensen, W. L. J. Chem. Phys. 1982, 77, 4156

14. Darden, T.; York, D.; Pedersen, L. J. Chem. Phys. 1993, 98, 10089.

15. Ryckaert, J. P.; Ciccotti, G.; Berendsen, H. J. C. J. Comput. Phys. 1977, 23, 327.

16. Feller, S. E.; Zhang, Y.; Pastor, R. W.; Brooks, B. R. J. Chem. Phys. 1995, 103, 4613. 